\title{
Rockbursts Mud and Plastic
}

\author{
Nev Fowkes $^{\text {a }}$

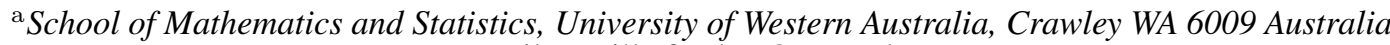 \\ Email: neville.fowkes@uwa.edu.au
}

\begin{abstract}
The gold mines in South Africa are the deepest in the world and rockbursts (including tunnel collapse) are a common occurrence, endangering life, causing damage to rock support structures and resulting in costly delays. Rockbursts are violent outbursts of broken rock and concrete in tunnels primarily caused by the accumulation of elastic strain energy in the tunnel walls but often triggered by seismic events. During a rockburst hundreds of tonnes of pulverized rock may be expelled into openings accompanied by a sudden sharp sound, an air blast, and a dense cloud of dust. The air blast itself can propagate kilometers through the tunnel system and be of sufficient strength to overturn vehicles.
\end{abstract}

Conventional wisdom is that rockburst activity is much less likely in tunnels with water or mud on the floor. We examine mechanisms that may explain such reduced activity using simple mechanistic models. The investigations suggest that lubrication effects due to the presence of water within cracks could inhibit, and in some cases prevent, the expulsion of rock lumps from tunnel walls. The effect of the water in a crack is to produce a suction force proportional to $\mu \omega / h_{0}^{\alpha}$ with $\alpha \approx 5$, where $h_{0}$ is the crack thickness, $\omega$ is the frequency of the impacting earthquake wave and $\mu$ the shear viscosity of water. Thus strong suction forces occur for small cracks and high frequency impacting earthquakes. These forces may cause an almost detached rock lump to remain attached, or detach if the eruption time span is large enough and the attachment to the walls is weak enough. Estimates suggest that both scenarios are possible in practice. These results also suggest that coating the tunnel walls with moisture containing semi liquid pastes or spray on plastic materials may be effective for tunnel wall stabilization.

Keywords: Rockbursts, mining tunnel collapse, crack repair, lubrication models 


\section{INTRODUCTION}

Mines are often located near fault planes and the stress redistribution due to slip may induce rockbursts in nearby tunnels. Also the stress waves generated by such events may trigger slip on other remotely located fault planes, again resulting in rockbursts. Mining induced movements caused by tunnel digging, mechanical vibration or blasting can also trigger rock blast events locally or remotely. Typically there are hundreds of such seismic events each year directly related to mining activities, most of which are minor. One would like to anticipate and hopefully avoid, or protect against, such events.

Mathematics in Industry Study Group meetings have been held in South Africa yearly since 2004 and almost every year a problem related to rockbursts has been presented for consideration of the group. Some of the problems presented to the MISG have been related to the above tectonic or mining sources, see for example Fowkes, Mason and Napier (2004), and Napier (2006). Other MISGSA investigations have been concerned with the followup effects of a rockburst, for example attempts have been made to model the airblasts generated by rockbursts and tunnel collapse, see Mureithi et al (2005) and Kalique et al (2006). Still other studies have been concerned with effective defense of walls against an impacting stress wave. The present problem is associated with wall defense. Similar studies have also occurred in the Australian and New Zealand context, see for example Marchant and Weir (2004).

Structural support for the tunnel walls can be provided by a thick layer of shotcrete (concrete). The thick and strong arched walls transfer stress from the walls to the floor of the tunnel. However, more surprisingly, thin and relatively elastically weak spray-on liners (TSLs) have also been found to be effective for stabilizing the rock walls of mining tunnels, see Potvin, Stacey and Hadjigeorgiou (2004). Such liners are sprayed onto the rock surface with a typical thickness of 4-mm and are very flexible (Young's modulus E typically $0.2 \mathrm{GPa}$ ) compared with rock (E typically $70 \mathrm{GPa}$ ), so that structural support is not the mechanism. The suggested mechanism in this case is related to crack extension. The sprayed on TSL's are thought to penetrate and effectively repair surface cracks so that the fracturing required for rock detachment doesn't occur. Theoretical results show that the effect of the sprayed on material (providing it penetrates the crack) is to change the stress singularity at crack tips, and almost complete repair (no stress singularity) occurs even with elastically weak TSL's, see Fowkes and de Freitas (2008). Another advantage of TSL's is that they are inexpensive to apply.

As indicated above it has been observed that rockburst activity seems to be less in tunnels with water or mud on the tunnel floor. The objective presented to the MISG was to investigate various mechanisms that could explain this. The 'evidence' is anecdotal and is not supported by any hard facts (for example on site statistical data). Furthermore there is almost no data concerning the local structural and physical environment involved in associated circumstances. In mining engineering such a lack of data is common; the environment is challenging. It is not even clear if the mud on the floor is responsible for the apparent changed behaviour or if the presence of the mud simply indicates wet conditions which may significantly effect behaviour; so mud is simply a symptom not the cause. A number of wave propagation mechanisms were briefly investigated. A positive pressure pulse propagating through rock is reflected at a free surface as a tensile pulse, and because rock is weak under tension, the surface may rupture causing a rockburst. One thought was that the presence of a mud layer on the rock face may alter the strength of the tensile pulse at the wall surface (the $\mathrm{P}$ and $\mathrm{S}$ wave composition is altered) and thus effectively cushion the rock face. A simple model showed that the effect is small, see Fowkes and Mureithi (2008). Another thought was that refraction effects associated with variations in moisture content in the rock around the tunnel may lead to a redirection of waves away from the tunnel face. Water is often used to settle dust so that the tunnels are often wet. Investigating this required more information about the moisture distribution than available, so no conclusions were drawn. In the end it was felt that it was most likely that crack propagation issues may again provide a possible explanation for reduced rockburst activity, with water as the agent of change in this case.

\section{A LUbrication MOdel}

Naturally occurring rock is heavily fractured as a result of past crustal movements. The effect of an impulse can be to rupture remaining connections between an individual block of rock and the surrounding rock wall so that this block of rock is ejected from the wall, floor or ceiling. Furthermore the structural 
integrity of the tunnel can be compromised as a result of the dislodgment, so that the tunnel may collapse. Water present in the tunnel may penetrate into the thin fractures (gravitationally and/or surface tension driven) and the resultant thin lubricating layer may provide strong resistance to separation between the rock and the surrounding wall. In this way the rockburst may be avoided. To assess this effect we examine a simple lubrication model, see Figure 1.
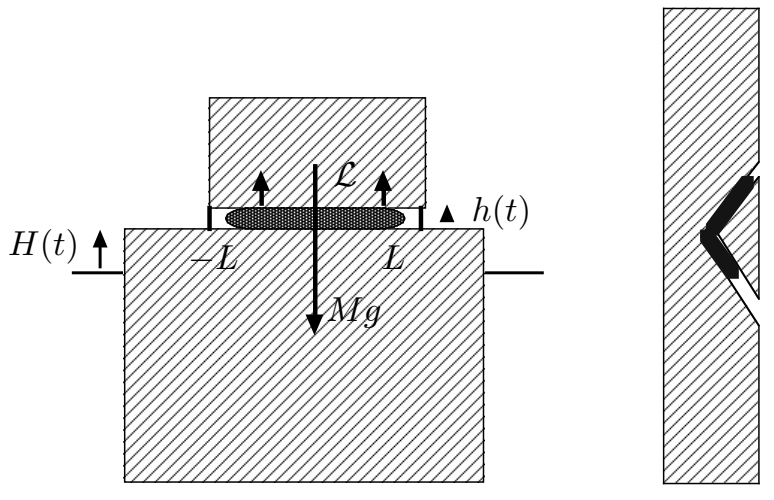

Figure 1. Left: the oscillating table model. Right: a wall rockburst.

In this model we have a mass $M$ supported by (weak) elastic springs on a table. Under equilibrium conditions the gap between the table and the mass is $h_{0}$ (so $M g=k h_{0}$ ). The table oscillates with frequency $\omega$ and amplitude $H_{0}$, so that

$$
H(t)=\bar{H}+H_{0} \sin \omega t
$$

where $H(t)$ is the height above a prescribed datum. If the oscillation is sufficiently vigorous the supporting 'springs' will break and the weight will not return to its resting position. If however there is a thin lubricating layer of water (thickness $h(t)$ ) and length $2 L$ and fixed depth $Z$ (into the page)) separating the mass from the table, then the suction generated within this layer may reduce the motion sufficiently to prevent separation. The mass represents a rock lump which is tenuously attached to the table (the rock floor, tunnel wall or roof) by weak elastic springs which model the intact connections between the rock lump and the solid tunnel. The gap (or crack) between the rock (mass) and the wall is filled with water. The surface area of contact $S$ between the water layer and the two rock faces corresponds to a contact of length $2 L$ and fixed width in the table model. Since the depth $Z$ is fixed in the table case the water layer will only expand and contract in the $x$ direction; the flow will be unidirectional. In practice the rock will be 'wedge shaped' and the gap will not be uniform and the flow will be more cylindrical. Full contact between the rock faces will occur at isolated resting points or points of attachment (the elastic springs); we assume the flow will not be greatly hindered by such obstructions. The length scale of impacting waves is generally much larger than the dimensions of tunnel, let alone the rocks that break away from the tunnel walls or floor, so that the impinging wave is accurately modeled by a wall (or table) oscillation.

The equation of motion of the mass (rock) is:

$$
M(\ddot{H}+\ddot{h})=\mathcal{L}-F_{0}
$$

where the elastic restoring force $F_{0}$ is given by

$$
F_{0}=k h(t)-M g=k\left(h_{0}-h\right),
$$


where $h_{0}=M g / k$ is the equilibrium gap size, and where the quasi-steady total upward force due to the lubricating layer is given by

$$
\mathcal{L}=\int_{S} p(x, y, t) d x d y \quad\left(\mathcal{L}=Z \int_{-L}^{L} p(x, t) d x \text { in the } 1 D \text { flow case }\right)
$$

$p$ is the pressure exerted by the water layer on the mass. These equations model the movement of a rock attached to either the floor, the walls or the roof of the tunnel (as in Figure 1 Right). In the 1D table model depicted in Figure 1, $F_{0}=M g$ but of course its more likely that rock will detach from the walls or ceiling. Also in this $1 \mathrm{D}$ case $\mathcal{L}=S \int_{-L}^{L} p(x, t) d x$. For structural reasons detachment from the supporting walls is more likely.

\section{The lubricating layer force}

The effect of the oscillation is to cause the water layer to be sucked backwards and forwards between the rock faces generating the lubrication force on the rock mass as it does so. The total volume $V_{0}$ of the lubricating layer remains fixed. For simplicity we will consider the situation in which the gap under the rock is uniform and horizontal.

The (quasi-static) pressure distribution within the lubricating squeeze layer in the general case is governed by Poisson's equation

$$
p_{x x}+p_{y y}=\left[\frac{12 \mu}{h^{3}} \frac{d h}{d t}\right] \text { in } \mathcal{S},
$$

with $p=0$ around the boundary $\partial \mathcal{S}$, where $\mathcal{S}$ is the 'surface' of water/rock contact; $\mu$ is the shear viscosity of water. In the fixed depth lubricating layer table case of Figure 1 Left (the parallel flow case)

$$
p(x, t)=-\frac{\left(x^{2}-L^{2}\right)}{2}\left[\frac{12 \mu}{h^{3}} \frac{d h}{d t}\right], \text { so that } \mathcal{L}=-\frac{2}{3} Z L^{3}\left[\frac{12 \mu}{h^{3}} \frac{d h}{d t}\right],
$$

and since the volume of water $V_{0}=Z(2 L(t)) h(t)$ remains fixed (with $Z$ also fixed) we see that

$$
\mathcal{L}=-\frac{\mu \dot{h}(t) V_{0}^{3}}{Z^{2} h^{6}(t)}=-K_{1} \frac{\dot{h}(t)}{h^{6}(t)} \text { (say); }
$$

it is this very strong dependence of the lubrication force on the layer thickness (for small thicknesses) that underlies the physics of lubrication. In the purely cylindrical flow case $p(x, y, t)=p(r, t)$ and (5) integrates to give

$$
p(r, t)=-\left(r^{2}-R_{0}^{2}\right)\left[\frac{12 \mu}{h^{3}} \frac{d h}{d t}\right] \text { so that } \mathcal{L}=-\frac{\pi}{8} R_{0}^{4}\left[\frac{12 \mu}{h^{3}} \frac{d h}{d t}\right],
$$

where $R_{0}$ is the radius of the water layer. In this case $V_{0}=\pi R_{0}^{2} h(t)$, so that

$$
\mathcal{L}=-\frac{3 \mu \dot{h}(t) V_{0}^{2}}{2 h^{5}(t) \pi}=-K_{2} \frac{\dot{h}(t)}{h^{5}(t)}(\text { say })
$$

significantly the dependence of the strength of the lubrication force for small layer thicknesses is reduced from that obtained in the unidirectional flow case because the flow is then less constrained. Both situations are likely to occur in practice. We will work with the unidirectional flow case here; the cylindrical flow case results are very similar.

\section{Parameter values}

For present estimates we will consider a cylinder of granite of radius $20 \mathrm{~cm}$ and thickness $15 \mathrm{~cm}$. Such a rock would weigh $52 \mathrm{~kg}$ (the density of granite is $2.75 \mathrm{gm} / \mathrm{cm}^{3}$ ) and its dislodgment from a tunnel wall could well cause a significant rockburst. If the induced suction force to weight ratio

$$
\left|\frac{\mathcal{L}}{(M g)}\right|=\frac{3 \mu \dot{h}(t) V_{0}^{2}}{2 h^{5}(t) \pi M g}
$$


for this rock is of unit order then one would expect lubricative forces to significantly effect dislodgment (dynamic details later). The strength of the lubricative force increases linearly with rock speed and dramatically with gap thickness $h$. If we take rock speed as $1 \mathrm{~m} / \mathrm{sec}$ as being typical of mining induced rock wall speeds and a gap of $1 \mathrm{~mm}$, then this ratio is 14.8 ; i.e. lubrication forces much larger than gravitational forces are possible! Seismic induced rock particle velocities are likely to be more of the order of $10 \mathrm{~cm} / \mathrm{sec}$; for such speeds the ratio is of unit order. Cracks smaller than $1 \mathrm{~mm}$ are perhaps more usual, so that lubricative forces can be much larger, although the attachment force (modelled by the spring) is also likely to be greater for thin cracks. The conclusion is that if water is present within the cracks then lubricative forces will certainly be significant.

Returning to the dynamics of the semi-detached block's motion as contained in (2) it is it is convenient to introduce the scales

$$
t=t^{\prime} / \omega, H=H_{0} H^{\prime}\left(t^{\prime}\right), \text { and also } h=H_{0} h^{\prime}\left(t^{\prime}\right) .
$$

It should be appreciated that the amplitude of the vibration would be normally much greater than the water layer thickness, so this choice is something of a geometric/physics compromise; the lubrication force is very strongly gap size dependent and so the size of the above coefficient underestimates $\mathcal{L}$. Working with the $1 \mathrm{D}$ flow case this leads to the dimensionless description

$$
\ddot{h}+\eta\left(h-h_{0}\right)+\xi \frac{\dot{h}}{h^{6}}=\sin t \text { in } t>0,
$$

where primes have been dropped and where the dimensionless groups are

$$
\eta=\frac{k}{M \omega^{2}} \text { and } \xi=\frac{K_{1}}{M H_{0}^{6} \omega} .
$$

The parameter $\eta$ is the ratio of the natural frequency of vibration associated with the rock wall/rock (spring) attachment to the frequency of vibration of the table. We will assume the rock is weakly attached so that $\eta \ll 1$. The parameter $\xi$ measures the ratio of the lubricative force on the mass to the force exerted by the table movement; as indicated earlier this is strongly dependent on the initial gap size. For purpose of illustration we will use $\eta=0.01$, and $\xi=0.1$. We examine the situation in which

$$
h(0)=h_{0}, \text { and } h^{\prime}(0)=1,
$$

so that the mass (rock) is initially moving with the table (wall). Given the above parameters we expect lubricative forces to be relatively small for $h_{0}$ of unit order and becoming larger as $h_{0}$ reduces; the initial layer thickness $h_{0}$ thus provides a useful parameter for displaying the effect of gap thickness on the dynamics. In the absence of the lubricating layer the mass will immediately separate from the table moving with the initial table (or wall) speed, however eventually (with this spring model) the restoring force will return the mass to its equilibrium position. In practice the connection may be broken and the rock will dislodge. This can be seen in Figure 2 for $h_{0}=1,2$ where the table (wall) position and rock trajectory are plotted; evidently the lubricating layer has very little effect for such gap sizes. For small values of $h_{0}\left(h_{0}<0.3\right)$ the rock will remain 'attached' to the wall, see Figure 2 (Right). For values of $0.5>h_{0}>0.3$ one sees an evolving behaviour in which the lubrication layer partially prevents separation. The detailed behaviour in this range depends on the relative size of the restoring force as expressed in $\eta$. Evidently detachment may not occur within this gap range if the oscillation is not sustained; a detailed analysis will not be undertaken here.

\section{Conclusions}

It was found that suction forces due to the presence of a water layer in rock cracks can be very large for thin cracks and this will act to inhibit rockburst damage. This could well provide an explanation for the observed effect of moisture on rockbursts. Of course rockbursts are spasmodic so checking out this explanation in the field is not a trivial matter, however, it is a simple matter to experimentally check out the mechanism described; our experience with lubrication engineering suggests that the mechanism will work if indeed water fills the cracks. Under such circumstances the main issue is whether water will penetrate into cracks and will remain there. 
Nev Fowkes, Rockbursts mud and plastic

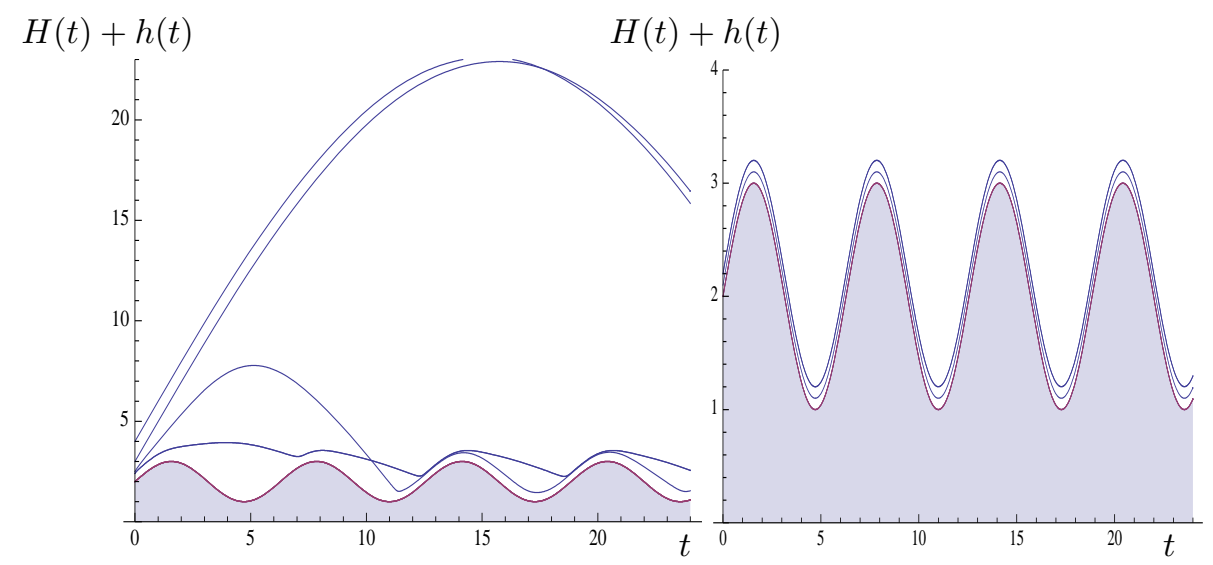

Figure 2. Lubrication Effects: the displacement $h(t)+H(t)$ of the rock under the action of different thickness water layers. Left: $h_{0}=0.3$ (bottom curve), 0.4, 0.5, 1, 2 (top curve). Right: $h_{0}=0.1$ (bottom curve), $0.2,0.3$ (top curve, with expanded vertical scale). The rock face (or table) position $H(t)$ is shaded. $(\eta=0.01, \xi=0.1)$

This work raises the possibility of using a semiliquid paste to stabilize tunnel walls. It has been noted earlier that solidified spray on plastics (TSL's) have been shown to be effective for wall stabilization, so a semi-liquid paste may combine both beneficial features. Studies are being undertaken to investigate this.

\section{Final REMARKS}

Mining engineering is as much an art as a science. There are many reasons for this. The displacements are large, as much plastic as elastic, and highly variable. Additionally the mechanical properties are as much determined by the cracks as the solid rock material. Also normally the aim is not so much to limit displacement size in tunnel walls as to reduce stress build up by allowing large movements. This means that classical engineering experience and mechanical models are of limited applicability. Added to all this is the lack of available on site data. Nevertheless much progress has been made. Mining accidents are remarkably rare given these circumstances, so engineers have learnt how to cope with this uncertainty. It is hoped that improved models will be developed in the future, but a new approach is probably required. For a start most of the available data is seismic data so correctly interpreting such data is vital.

\section{REFERENCES}

Fowkes,N., de Freitas, J. and T. R. Stacey (2008). Crack repair using an elastic filler. Journal of the Mechanics and Physics of Solids. 56, pp. 2749-2758.

Marchant, T. and G. Weir (2004). Earthquake damage in underground roadways. Proceedings of the Maths in Industry Study Group NZ 2004, ed. G. C. Wake, pp. 89-102.

Mason, D. P. and T.R. Stacey (2005). Support to rock excavations provided by thin sprayed liners. In Proceedings of the 2005 Maths in Industry Study Group South Africa, ed. D. P. Mason., pp. 77-105.

Napier, J. A. L. (2006). Analysis of the potential mechanisms of rockbursts. In Proceedings of the Maths in Industry Study Group South Africa 2006, ed. D. P. Mason, pp. 9-19.

Kalique, M., Anthonyrajah, M. Mason, D. P., Mureithi, E. and J. R. Stacey (2006). Modelling airblasts in a long tunnel with surface roughness. In Proceedings of the Maths in Industry Study Group South Africa 2006, ed. D. P. Mason, pp. 77-108.

Mureithi, E. and Sjoberg, Stacey, T. R. and J. R. Ockendon (2005). Piston effect due to rock collapse. In Proceedings of the Maths in Industry Study Group South Africa 2005 ed. D. P. Mason, pp. 17-39.

Mureithi, E. and N. Fowkes, (2008). Rockbursts and mud. In Proceedings of the Maths in Industry Study Group South Africa 2008 ed. D. P. Mason, pp. 27-41. 
Nev Fowkes, Rockbursts mud and plastic

Stacey, T.R. (2001). Review of membrane support mechanisms, loading mechanisms, desired membrane performance, and appropriate test methods. Journal of the South African Institute of Mining and Metallurgy, 101, pp. 343-351.

Stacey, T.R. and X. Yu (2004). Investigations into mechanisms of rock support provided by sprayed liners. In Ground Support in Mining and Underground Construction. ed. E. Villaescusa and Y Potvin, pp. 563-569. 